\title{
Arguments for inclusion of economic criteria in the total maximum daily load (TMDL) allocation phase
}

\author{
Arjumand. Z. Zaidi $^{1} \cdot$ Sharon. M. deMonsabert ${ }^{2}$
}

Received: 25 June 2015/Accepted: 31 August 2015/Published online: 15 September 2015

(C) Springer International Publishing 2015

\begin{abstract}
The idea of including economic analysis in pollution control studies is not new. There is a long tradition of research modeling least cost load allocation for water pollution control that has led to a substantial awareness that failure to take costs into account in load allocations can greatly increase the costs of water pollution control. Despite this understanding, the current load allocation approach adopted under United States Environmental Protection Agency total maximum daily load (TMDL) program allocates pollutant loads among different polluters in the absence of economic criteria. Many water quality professionals question the appropriateness of including economic criteria into an already complex and cumbersome TMDL allocation process. The question is not why we do not include economics into waste load allocations, rather the question is why we should? This paper attempts to identify and present the benefits associated with the incorporation of economic criteria into the traditional TMDL allocation process. The TMDL process is
\end{abstract}

Arjumand. Z. Zaidi

azaidi@gmualumni.org; arjzaidi@gmail.com;

arjumand.zaidi@ist.edu.pk

Sharon. M. deMonsabert

sdemonsa@gmu.edu

1 Institute of Space Technology (IST), SUAPRCO Road, Sector 28, Gulzar-e-Hijri, Off University Road, Karachi 75270, Pakistan

2 Civil, Environmental and Infrastructure Engineering, George Mason University, Fairfax, VA 22030-4444, USA considered to be a holistic watershed approach and as such should not overlook the economic consequences of pollutant allocation alternatives. This paper attempts to focus less on the mechanism of optimizing the costs and more on the benefits derived as a by-product of doing so. The advantages of incorporating economic criteria for a bacteria load allocation in the Roses Creek watershed in the Commonwealth of Virginia are presented in this paper.

Keywords Best management practices · Economic factor - Optimization model - Water resources . Watersheds · Water quality

\section{Introduction}

The United States Clean Water Act (CWA) section 303(d) requires states to identify impaired water bodies and develop a watershed plan which includes the establishment of a total maximum daily load (TMDL) on a priority schedule (USEPA 2014). The current approach, mandated by the United States Environmental Protection Agency (USEPA), allocates pollutant loads among different polluters in the absence of economic criteria. The Protocol for Developing Pathogen TMDLs (USEPA 2001) recommends considering cost-effectiveness for allocations to achieve a balance between waste load allocations (WLAs) and load allocations (LAs). Currently, economic analyses are performed at a later stage once the loads have been allocated and various implementation strategies are under evaluation. This approach to water quality management results in sub-optimal and in some cases, infeasible load reduction allocations. An economically superior allocation, that satisfies all water quality goals, may offer a preferred solution. 


\section{Proposed approach}

A TMDL allocation methodology that considers abatement costs at the load allocation phase has been developed by Zaidi and deMonsabert (2003), Zaidi (2004), deMonsabert and Zaidi (2005), and Zaidi et al. (2008). This is called Economic TMDL model that minimizes costs associated with pollutant load allocations. The schematic diagram of the existing approach to the development and allocation of pollutant loads is shown in Fig. 1. In the existing approach, following the listing of the impaired waters, a prioritized list is developed for load allocation. Point source and nonpoint source loads are determined using the available data and information and through field studies. Load allocations are done using any hydrologic model. Water quality models are used to evaluate the impact of the estimated loads on the waterbody.

The effectiveness of the TMDL implementation may be evaluated through water quality monitoring. Unfortunately, this happens for the first time at the implementation stage of the TMDL process after the load allocations have been established. The proposed methodology incorporates not only the economic efficiency into the

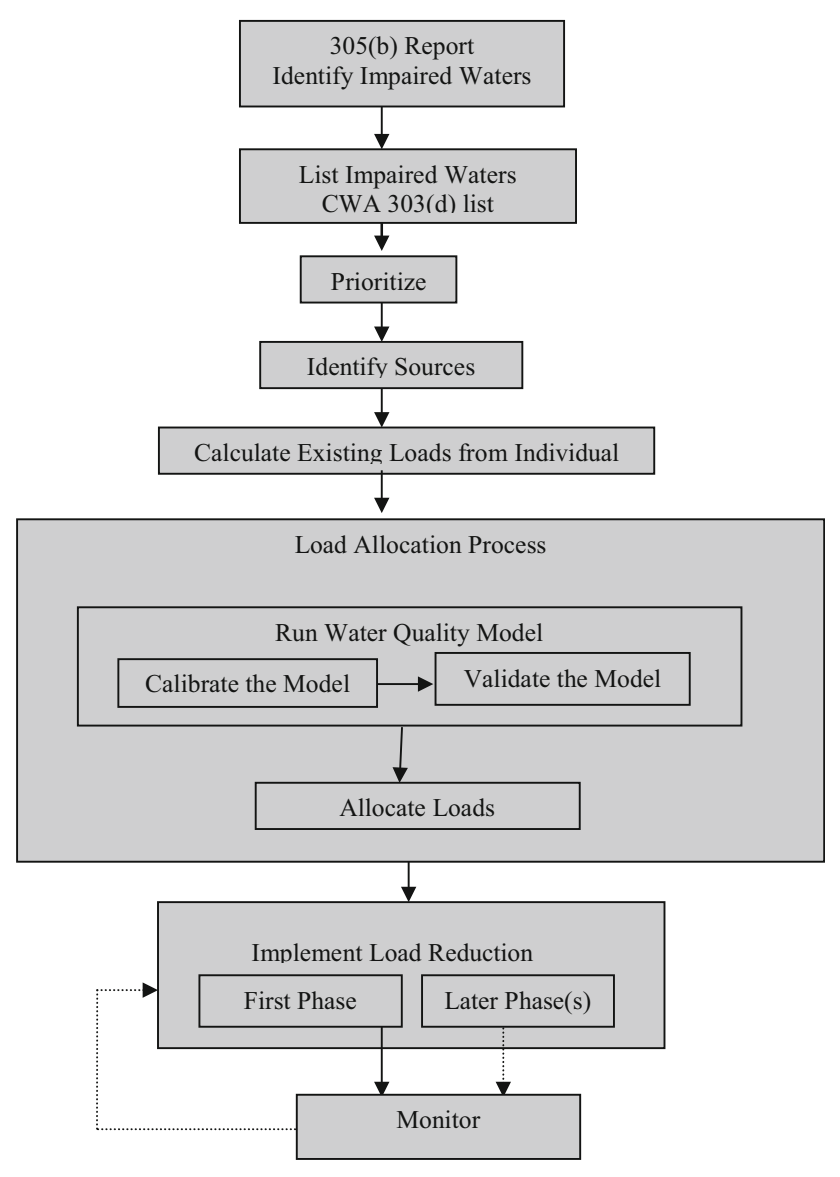

Fig. 1 Total maximum daily process (current approach) load allocation phase of the TMDL process (see Fig. 2) but also checks the technical feasibility of the available control measures. Following the water quality model calibration and validation, an economic optimization model appropriate to the watershed, is developed. This model calculates the minimum cost feasible implementation strategy for a given load at a watershed level. The model can also minimize the cost of alternate allocation schemes to provide more flexibility to the decision makers if due to some other reasons the least cost allocation scenario is not acceptable to them.

\section{Feasibility of a TMDL allocation}

The feasibility of accomplishing a TMDL allocation is dependent on two factors: (1) the control measures' effectiveness and (2) the water quality standards applicable to the primary use classification. If the control measures are not capable of achieving the necessary load reduction, the allocation is infeasible. Similarly, if the water quality standards are higher than what can be achieved with existing technology, the allocation is still infeasible. The pollutant reduction levels as required by the TMDL may not be realistic; it is imperative that the practicality of the measures to achieve the levels is to be evaluated. Otherwise, an infeasible waste load allocation may only be identified as "infeasible" during the implementation phase. At this point, few options exist but to reallocate the loads that is a time consuming and costly impediment to the water quality improvements process.

\section{Limitations of control measure efficiencies}

Although the Best Management Practices (BMPs) are used to reduce pollutants, the effectiveness of BMP or BMP system is not always easy to predict. This is particularly difficult in the case of nonpoint source pathogen management. There is an inherent uncertainty regarding the level of pollutant reduction through the management options available in the literature for bacteria removal. Without better knowledge of the control measures' efficiencies, there is no assurance of achieving the water quality goals as described by the TMDLs.

The consideration of the available control measures' effectiveness during the TMDL allocation phase may also reveal the limitations of the TMDL allocations to meet the required state standards. For example, a random check of the EPA approved Virginia TMDL allocation reports for E-coli reduction (DEQ 2010) reveals that during year 2007 and 2008, the maximum reduction levels from different sources range from 90 to $100 \%$. These sources include direct/indirect livestock waste, 
Fig. 2 Total maximum daily process (proposed approach)

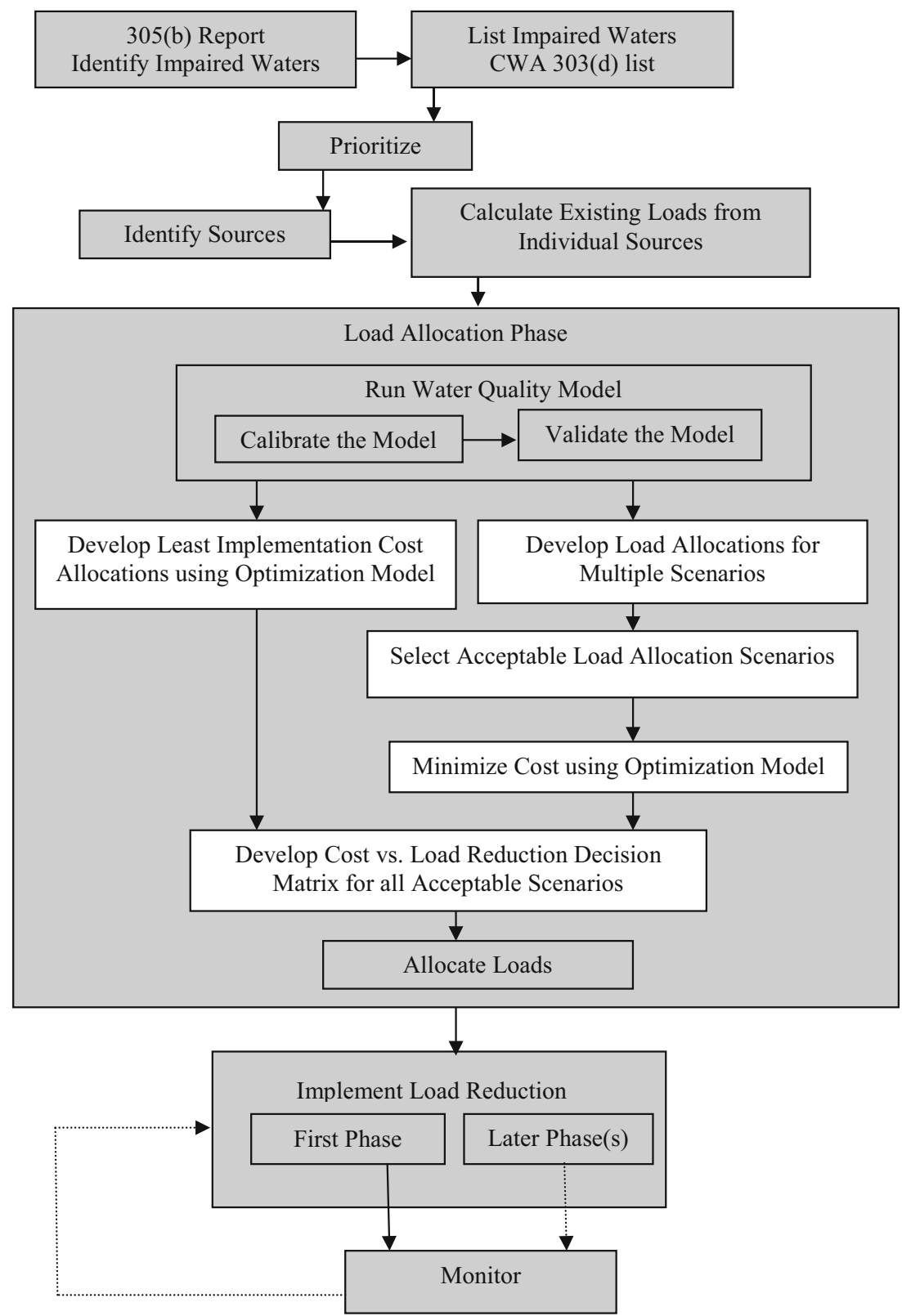

load from residential area, illegal (illicit) discharges, and direct inputs of wildlife fecal material to streams. To achieve such high level of reductions, it is necessary to evaluate the technical and economic feasibility of the available load reduction measures in order to ensure a successful TMDL implementation.

Estimation of the pollutant removal efficiencies of nonpoint source BMPs is still an evolving area of study. The results of such studies available in the literature are highly variable mainly due to the lack of data for the practices and varying site conditions. Therefore, there is an urgent need to further investigate the control measure performance and pollutant reduction effectiveness.

\section{Attainability of water quality standards}

The first advantage in incorporation of an economic analysis into the load allocation derivations is the ability to evaluate the attainability of the water quality standards for a suggested load allocation scenario. Modeling the costs and effectiveness of the BMPs will demonstrate if the standards can be attained with current technology. If the model yields an infeasible result, the standards cannot be achieved with even the most costly remediation strategy. For example, a $99 \%$ reduction in nonpoint bacteria load is not attainable with current BMPs. Buffer strips and other recommended alternatives can at best reduce nonpoint agricultural waste by 55-95 \% (Coyne et al. 1998, Young et al. 1980). 
During the investigation of the Roses Creek watershed TMDL, it was observed that the upper percentile water quality criterion adopted by Commonwealth of Virginia for E. coli TMDL was unattainable with existing BMPs. Upon further inspection, the nonpoint agricultural waste reductions for a majority of the state's TMDLs are similarly unattainable. The results of this research showed that the available pollutant control options for pathogen removal in an agricultural watershed (other than some of the source control measures that are not always feasible and may cause alteration of the land use practices of the watershed) are incapable of achieving the level of reductions required by the water quality standards. In the case of the Roses Creek watershed, modeling the effectiveness of the BMPs showed that the upper percentile E. coli criterion (235 counts/ $100 \mathrm{ml}$ ) was exceeded $40 \%$ of the time based on the maximum achievable effectiveness of these control measures. Also, the single sample maximum fecal coliform concentration was exceeded $38 \%$ of the time (only $10 \%$ is allowed) from the state-defined criteria of 400 counts/ $100 \mathrm{ml}$. Therefore, the proposed model gave an infeasible solution when run to satisfy all the given state standards. It is concluded, therefore, that the existing standards are unattainable for the Roses Creek watershed. To demonstrate the proposed approach, the water quality criteria were made less stringent by omitting upper percentile $E$. coli and single sample maximum fecal coliform concentration criteria from the optimization model and the model is run to meet 30-day geometric mean criteria for $E$. coli concentration. All the results of the economic TMDL analysis for the Roses Creek watershed presented in this paper are based on the less stringent water quality standard.

One advantage of modeling BMP effectiveness and associated costs is that the trade-off between the cost and the standard can also be evaluated through the proposed methodology at the watershed level. For example, the proposed model may be run several times by changing the value of the optimization constraint defining the water quality criteria. The minimum cost for each value of water quality standard can be calculated by the model and the results of optimization may be plotted against their respective water quality standards (see Fig. 3). Figure 4 illustrates the relationship between the water quality standard (30-day geometric mean in-stream E. coli concentration) and the attainment cost for the Roses Creek watershed.

\section{Additional benefits}

The likelihood of a successful TMDL implementation depends on a realistically attainable load distribution among the polluters. An analysis that relates the load reduction of the implemented BMPs to the water quality is needed to

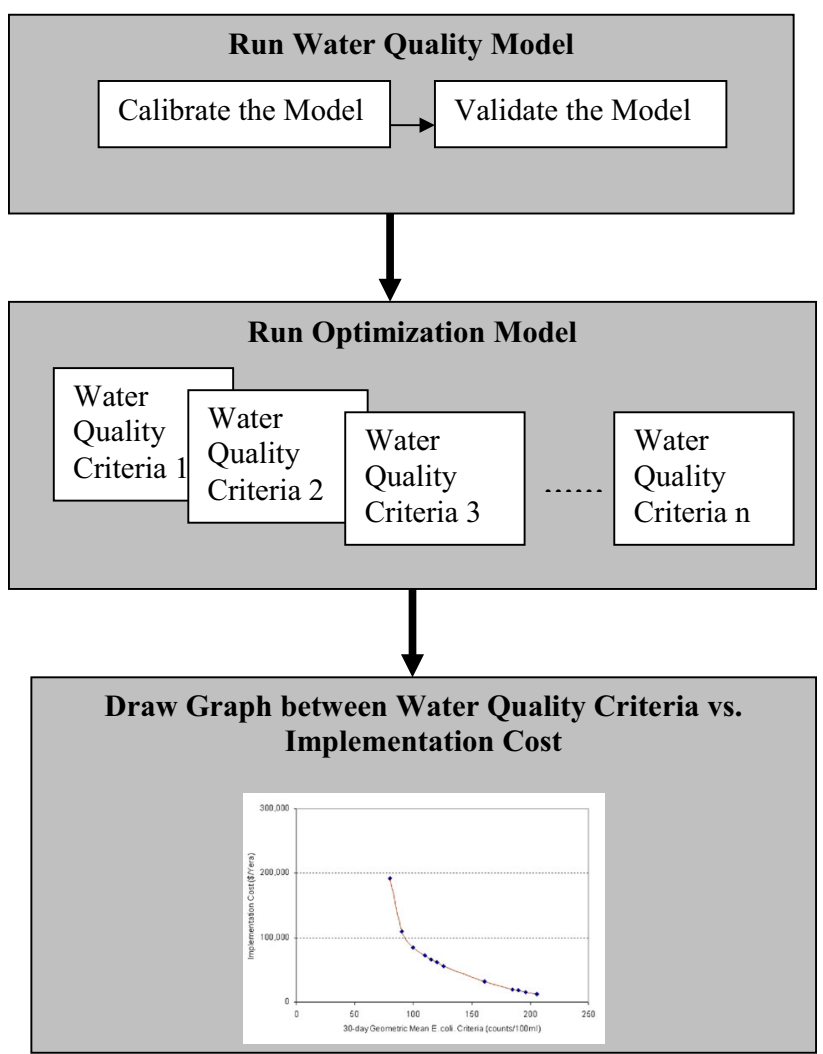

Fig. 3 Minimizing implementation cost at varying water quality criteria utilizing the proposed approach

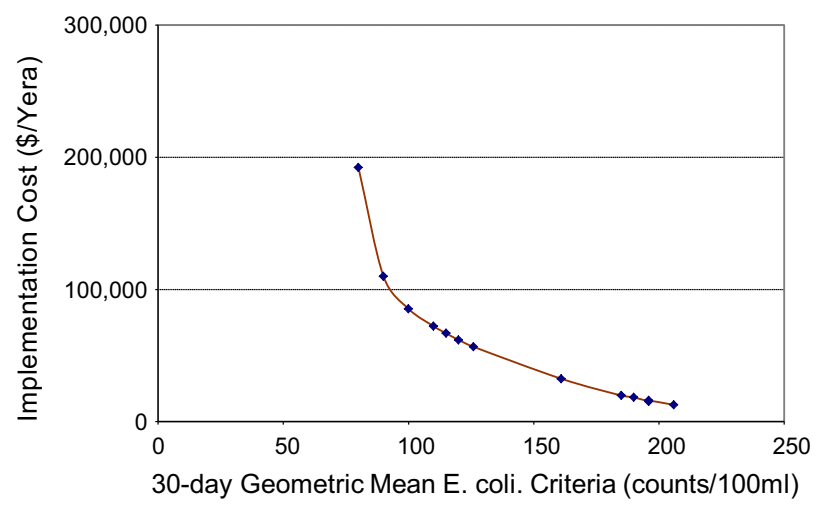

Fig. 4 30-Day geometric mean E. coli. criteria vs. implementation costs for the Roses creek watershed

ensure that the load allocation is feasible and will achieve the desired objectives. Incorporation of a mathematical model as demonstrated by Zaidi and deMonsabert (2003), Zaidi (2004), deMonsabert and Zaidi (2005), and Zaidi et al. (2008) will yield a solution that is technologically feasible as well as economically superior. The proposed approach will overcome many of the shortcomings of the current TMDL process. Advantages of utilizing the proposed framework include the following: 
- provide added flexibility to the TMDL decision makers to make informed decisions by improving the economic efficiency of the TMDL allocations,

- reduce the chances of costly revisions to the allocated loads during the implementation phase,

- may enhance public and stakeholders acceptance if allocation costs are known and minimized,

- encourage the use of a pollutant trading process if so desired through readily available cost information, and

- support phased implementation by providing information regarding the cost versus water quality criteria trade-offs.

\section{Other benefits beyond TMDL}

Although the specific use of this model has been demonstrated on the USEPA TMDL program, there are other benefits that extend beyond the TMDL program. The proposed approach can be utilized for minimizing the cost of pollutant remediation efforts in severely economically constrained regions of the world. Public health concerns all over the world would drive other countries to adopt similar techniques. The coupling of environmental remediation actions and cost will greatly improve the ability to successfully implement any restoration program anywhere in the world.

\section{Conclusions}

This paper presents the benefits associated with incorporation of an economic assessment into the allocation phase of the TMDL process. Cost minimization may provide a rationale in support of an economically acceptable load reduction allocation scheme among many polluters. It is not always necessary that the least cost scenario is preferable to the TMDL decision makers. In such cases, alternative load allocation scenarios with associated cost trade-offs may be fully understood. The economic analysis during the TMDL allocation phase allows the watershed planners and stakeholders the opportunity to make informed decisions while knowing the costs of the tradeoffs. Also, the alternate TMDL allocation scenarios may be evaluated utilizing the optimization process for the likelihood of cost-effective pollutant trading among pollutant sources in the watershed.

\section{References}

Coyne MS, Gilfillen RA, Villalba A, Zhang Z, Rhodes R, Dunn L, Blevins RL (1998) Fecal bacteria trapping by grass filter strips during simulated rain. J Soil Water Conserv 53(2):140-145

deMonsabert S, Zaidi A (2005) Is it really too hard to estimate TMDL allocation costs? In: Proceedings of third conference on watershed management to meet water quality standards and emerging TMDL, American Society of Agriculture and Biological Engineers, Atlanta, Georgia, pp 232-241, 5-10 March 2005

DEQ (2010) Virginia DEQ: TMDL development. Virginia Department of Environmental Quality. http://www.deq.state.va.us/tmdl/ develop.html. Accessed April 2010

USEPA (2001) The National Costs of the Total Maximum Daily Load Program. EPA-841-D-01-003; United States Environmental Protection Agency, Office of Water Washington, DC 20460, 1 Aug 2001. http://www.epa.gov/owow/tmdl/coststudy/coststudy. pdf. Accessed Dec 2009

USEPA (2014) Overview of impaired waters and total maximum daily load. US Environmental Protection Agency. http://water. epa.gov/lawsregs/lawsguidance/cwa/tmdl/intro.cfm. Accessed Dec 2014

Young RA, Huntrods T, Anderson W (1980) Effectiveness of vegetated buffer strips in controlling pollution from feedlot runoff. J Environ Qual 9:483-487

Zaidi A (2004) Economic TMDL allocation: an optimization framework. [Dissertation]. George Mason University, Fairfax (VA). Available from: University Database, Fairfax VA, Thesis Doctor I.T. 2004.Z35

Zaidi A, deMonsabert S (2003) Are your TMDLs cost effective? In: Proceedings of TMDL 2003 speciality conference at Chicago, Illinois, 16-19 Nov 2003, Water Environment Federation. Session 8, CD ROM, pp 858-868

Zaidi A, deMonsabert S, El-Farhan R (2008) How to include economic analysis in TMDL allocation. J Water Resour Plan Manag ASCE. 134(3):210-214 